\title{
Participación dialógica en la universidad: condición para el desarrollo del pensamiento crítico y el compromiso social
}

\author{
Héctor Monarca
}

\begin{abstract}
Resumen
Hace más de un siglo que las críticas a la escuela tradicional pusieron de relieve la importancia del aprendizaje activo. De la misma manera, con posterioridad, tanto desde el constructivismo como desde la psicología sociocultural se evidenció el papel relevante de la actividad de los estudiantes en sus aprendizajes. Finalmente, desde otros marcos teóricos como la sociología, la lingüística, la pedagogía crítica, etcétera, se ha insistido en la necesidad de la implicación de los estudiantes en sus propios procesos formativos. En esta línea, los aportes de Habermas y Freire han tenido importantes repercusiones en las prácticas de enseñanza, en donde lo dialógico se transforma en un rasgo y componente esencial de los procesos de construcción del conocimiento, del sujeto y de la sociedad. Este artículo aborda la participación dialógica de los estudiantes en la universidad, asumiendo un claro compromiso de ésta en la formación de profesionales críticos y comprometidos con la sociedad y el mundo en el que vivimos.
\end{abstract}

Palabras clave: participación dialógica; enseñanza universitaria; pensamiento crítico; compromiso social.

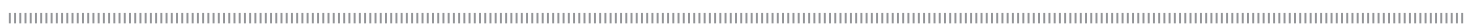

\section{Participação dialógica na universidade: condição para o desenvolvimento do pensamento crítico e o compromisso social}

\section{Resumo}

Faz mais de um século que as críticas à escola tradicional destacaram a importância da aprendizagem ativa. Da mesma maneira, posteriormente, tanto desde o construtivismo como desde a psicologia sociocultural evidenciou-se o papel relevante da atividade dos estudantes em suas aprendizagens. Finalmente, desde outros quadros teóricos, como a sociologia, a linguística, a pedagogia crítica, etecetera, insistiu-se na necessidade da participação dos estudantes em seus próprios processos formativos. Nesta linha, as contribuições de Habermas e Freire tiveram importantes repercussões nas práticas do ensino, quando o dialógico se transforma em uma caraterística e componente essencial dos processos de construção do conhecimento do sujeito e da sociedade. Este artigo aborda a participação dialógica dos estudantes na universidade, assumindo um claro compromisso desta na formação de profissionais críticos e comprometidos com a sociedade e o mundo em que vivemos.

Palavras chave: participação dialógica; ensino universitário; pensamento crítico; compromisso social. 


\begin{abstract}
Over a century ago the criticism of traditional schools highlighted the importance of active learning. Likewise, later on, constructivism and sociocultural psychology evidenced the relevant role of the students' activity in their learning processes. Last, other theoretical frameworks such as sociology, linguistics, critical pedagogy, etc., have insisted on the need to involve the students in their own educational processes. Along these lines, the contributions of Habermas and Freire have had important repercussions on teaching practices, where dialogical aspects are transformed into an essential feature and component of the processes to build knowledge, the individual and society. The present article addresses the dialogical participation of university students assuming the clear commitment of the university in the education of critical professionals committed to society and the world in which we live.
\end{abstract}

Key words: dialogical participation; university education; critical thinking; social commitment.

Recepción: 15/05/12. Aprobación: 13/11/12. 


\section{Introducción}

La participación ubicada como eje y como centro del aprendizaje y la enseñanza en la universidad supone asumir un papel como institución y como formadores en una clara conexión con la trasformación, la mejora y el cambio (Aguilera et al., 2010; Manzano y Torrego, 2009). Ubicar a la universidad en esta tarea es ir más allá de las demandas del mercado y de una acomodación de esta institución a las mismas; es ubicarla en los problemas y necesidades humanas y sociales, dentro de un marco público y contrastado de construcción de conocimientos; lo cual supone formar profesionales reflexivos y comprometidos con esta tarea (Giroux, 2003). Así, la universidad dentro de su función formativa específica, se ubica en relación con un tipo de sociedad a la que aspira, con un rasgo irrenunciable, la justicia social, una sociedad justa y democrática para la que forma y prepara a los sujetos.

Por otra parte, incluir a la participación como base para el desarrollo del pensamiento crítico y el compromiso social, y a éstos como objetivos de la formación universitaria, es asumir que la universidad también forma ciudadanos activos y profesionales comprometidos con la transformación y la justicia social. De esta manera, se vinculan las funciones de la universidad con la formación de profesionales reflexivos, comprometidos con la construcción de una sociedad participativa, democrática; profesionales con una clara conciencia de sus posibilidades y su responsabilidad de formar parte activa en los procesos sociales y educativos, como protagonistas activos, y no como meros espectadores en una sociedad de consumo.

Finalmente, la participación desde el punto de vista didáctico, supone tanto una forma de entender la enseñanza y el aprendizaje como un contenido en sí mismo de la formación. Desde este punto de vista, la participación se presenta como condición necesaria para el desarrollo del pensamiento crítico y del compromiso del sujeto con su entorno inmediato y con la sociedad en general. La participación también aparece como una forma de entender el conocimiento y su construcción.

En este artículo se abordan los argumentos que justifican el desarrollo de prácticas formativas basadas en la participación dialógica de los estudiantes universitarios. De la misma manera, se profundiza específicamente en el debate acerca de las posibilidades y las condiciones para un diálogo igualitario, mediante los aportes de Habermas, Apel, Freire y Dussel. Se entiende que ciertas formas de participación dialógica constituyen la base para la construcción del pensamiento crítico y el desarrollo de un sujeto comprometido con la sociedad.

\section{Fundamentos para el debate}

La participación de los estudiantes no es un tema nuevo dentro de la teoría de la educación. La misma ha sido abordada desde distintos marcos teóricos y con diversos intereses. Quizá haya sido el movimiento de la Escuela Nueva el que por primera vez lo pone de manifiesto de forma clara y evidente dentro de sus críticas al funcionamiento de la escuela tradicional (Carreño, 2002). En este caso, la crítica redimensiona el papel de la actividad del alumno en el aprendizaje y cuestionando su pasividad. Y aunque la mayoría de estas críticas se centraron en lo metodológico, también contamos con algunas referencias a la relación entre escuela-individuo-sociedad. Encontramos aquí a Dewey (1995) como un referente importante en esta dirección, quien, aunque no realiza un cuestionamiento al funcionamiento social, sí establece una importante conexión entre la escuela y la sociedad, dejando al descubierto la inexistente neutralidad tantas veces defendida. En una línea similar podemos ubicar los importantes aportes de Giner de los Ríos $(1916,1924)$ y de Luzuriaga (1968). 
Por otra parte, desde otros marcos interpretativos, encontramos las críticas hacia el autoritarismo escolar (Colmenar, 2002), las cuales pusieron de manifiesto las relaciones de poder existentes en el vínculo educativo basadas en la dominación y en la sumisión a la autoridad, también interpretadas como violencia simbólica y arbitrariedad cultural por Bourdieu y Passeron (1985).

Sin embargo, muchas de estas críticas han sido cuestionadas por el determinismo que subyace, al no dejar margen para las alternativas de los sujetos que quedan atrapados en las estructuras que los dominan, determinados por las mismas. Desde estas críticas, la construcción de lo social no es una mera producción o reproducción de los poderes dominantes, los sujetos no son meros espectadores o pasivos consumidores del devenir histórico (Giroux y McLaren, 1998). Estas posturas otorgan un valor irremplazable a la participación dialógica en los procesos de configuración de sujetos críticos y de construcción de lo social como proceso intersubjetivo.

Desde este punto de vista, las prácticas educativas basadas en el "ideal dialógico" son una oportunidad, en el sentido que ofrecen Giroux y MacLaren (1990) cuando hacen referencia a la necesidad de construir un espacio contrahegemónico, para una profunda y verdadera democracia. Aquí encontramos la importante tradición generada por Freire (2002, 2004), estos aportes ubican a los sujetos en una posibilidad transformadora de la realidad social y educativa, bajo nuevas prácticas pedagógicas basadas en un diálogo emancipador. Un diálogo cuyo sentido se encuentra en el "empoderamiento" de los sujetos, mediante el desarrollo de su conciencia como sujetos que se construyen a sí mismos y a la sociedad en la que habitan (Freire y Macedo, 1989). Desde este punto de vista, la participación es un acto social de intersubjetividad. Es, en sentido freiriano, un acto en el que se adquiere conciencia, una práctica social en la que los sujetos, a través del diálogo, toman conciencia de sí mismos y de la realidad en la que viven. Freire (2004) ve al debate y al diálogo como el método para hacer crítico al hombre, un espacio para la toma de conciencia de uno mismo, de sus circunstancias, de la sociedad en la que vive.

Vista así, la participación no es un mero acto individual, aislado, espontáneo; es asumir protagonismo en la construcción de la propia subjetividad (Freire y Macedo, 1989; Ramírez-Romero y Quintal, 2011) y en la acción educativa como acto social que tiene lugar en ese momento; en un contexto histórico, social y político concreto. Así, si la participación es parte del contexto, las características que asuman las clases de acuerdo con la propuesta didáctica, será otro de los factores clave para que la participación tenga lugar.

En este sentido, dar la voz tiene ya un valor en sí. Sin embargo, el salto está en la interacción entre lo subjetivo y lo objetivo, es decir, entre las experiencias del sujeto y los conocimientos socialmente construidos y la sociedad, sus estructuras de poder y de funcionamiento existentes. Interesa ir de la opinión a la argumentación, en este último caso, el sujeto se incorpora al proceso de construcción del conocimiento y de crítica social. En esta línea, Flecha (1997), al igual que otros autores ya mencionados, construye un concepto de diálogo igualitario bajo los supuestos teóricos que cuestionan las relaciones de poder y dominación; sostiene que se da en términos de igualdad cuando las aportaciones se tienen en cuenta por la validez de sus argumentaciones y no por la posición de poder que tiene el que las enuncia.

En relación con esto Young (1993) cuestiona ciertos pseudodiálogos caracterizados por el acomodamiento de las intervenciones de los estudiantes a las expectativas del profesor. Según este autor, la mala comunicación se da en un tipo de interacción que posee las siguientes características: 1) las respuestas que se buscan y estimulan no ayudan a 
la comprensión ni a la generalización, 2) no ayudan al desarrollo del pensamiento, 3) no estimulan la argumentación, 4) se basan más en el ajuste del alumno a la autoridad del maestro que en la veracidad de los argumentos, 5) no se le otorga estatus racional al alumno. Por eso, para este autor es clave analizar las preguntas que estimulan el diálogo, lo que ellas buscan, lo que incentivan.

Se emplea en esta dirección el término de "aprendizaje dialógico" (Elboj et al., 2002; Flecha, 1997), el cual hace referencia a una concepción comunicativa del aprendizaje que plantea que éste depende principalmente de las interacciones que se establecen entre las personas. En el caso de los grupos interactivos, la construcción de significados se basa en las interacciones que resultan de un diálogo igualitario entre los estudiantes, las familias y el profesorado (Elboj et al., 2002; Loroño, Alonso-Olea y Martínez-Domínguez, 2010).

Se observa así el valor que se le asigna al discurso y a los intercambios comunicativos como prácticas de construcción de lo social y de aprendizaje. Un gran número de autores enfatizan la dimensión semiótica del aula y la consideración de ésta como un escenario de construcción y negociación conjunta de significados. Así, por ejemplo, Coll y Onrubia (1996) hacen referencia a la potencialidad del discurso como instrumento para la construcción de significados compartidos en el aula, para ellos esto se debe a su naturaleza semiótica y a su capacidad para insertarse en la actividad conjunta que desarrollan los participantes.

En cualquier caso, la participación por medio de la palabra tiene una clara intención de empoderamiento de los sujetos; de formar profesionales reflexivos y comprometidos con el mundo, con la sociedad y la educación. En sintonía con esto Giroux y Mclaren (1998) sostienen que recién cuando damos voz a nuestra experiencia podemos transformar el significado que ésta tiene, mediante la reflexión crítica de los supuestos que la sostienen. En este marco, el reconocimiento de la responsabilidad hacia "el otro" hace que no sea un mero aplicador irreflexivo de consignas, tradiciones o prescripciones, sino que posiciona como un profesional que justifica su acción mediante una reflexión crítica colaborativa (Kemmis, 1999). En concreto, este último autor hace una referencia explícita al papel que tiene la reflexión en los procesos formativos.

Para Kemmis (1999) la reflexión crítica tiene las siguientes características: a) está orientada a la acción y forma parte de la historia, b) es un proceso social, c) es un proceso político, y d) es una práctica que expresa nuestro poder para reconstruir la vida social por la forma en que participamos en la comunicación, la toma de decisiones y la acción social. Siguiendo el pensamiento de este autor, pero intentando trasladarlo a una propuesta de formación universitaria, podemos decir que la reflexión debe orientarse a: 1) comprender la acción en contextos históricos concretos; 2) las relaciones sociales supuestas y prefiguradas en el pensamiento del actor y de la acción que adopte en razón de su reflexión; 3) los valores e intereses que enmarcan el pensamiento y la acción del actor en ese momento y contexto; 4) las bases ideológicas del pensamiento y de la acción para el actor en una situación concreta, y 5) la reflexión misma como una práctica en una situación concreta, históricamente configurada.

\section{Sobre la posibilidad del diálogo igualitario}

Como se ha visto en el apartado anterior, cuando se prioriza la participación reflexiva en los intercambios comunicativos del aula se está optando por una forma de entender la construcción del conocimiento y el papel que los sujetos tienen en este proceso. La base se encuentra en los argumentos y el contraste intersubjetivo que tiene lugar en los intercambios comunicativos y no, 
como se ha dicho, en la posición que los sujetos tienen en dichos intercambios. Sin embargo, esta afirmación debe ser problematizada, interrogada. Hablar de diálogo igualitario sin explicitar aspectos vinculados con el poder y la construcción y distribución del conocimiento en la sociedad actual, puede reforzar lo antagónico. Está claro que la posición que tiene un docente, aunque no siempre, en relación con las oportunidades que ha tenido para construir un determinado conocimiento, y el hecho efectivo de haberlo hecho, le otorga la "posibilidad privilegiada" de ofrecer una serie de argumentos. En este sentido, la participación no se ve al margen de los conocimientos que han sido apropiados por los sujetos. De esta manera, los conocimientos forman parte del proceso de participación, la cual ofrece y fomenta diversas posibilidades relacionadas con los procesos de objetivación y subjetivación del conocimiento.

Sin embargo, cuando se tiene a la participación dialógica como objetivo educativo dentro de los procesos formativos, atendiendo a las posibilidades que ésta ofrece para construir un razonamiento crítico, es necesario fomentar los procesos de indagación, de pregunta, de problematización del conocimiento y de los argumentos; asimismo, lleva a pensar por uno mismo en alternativas y puntos de vista. El razonamiento necesita de argumentos que obtenemos de los diversos campos de conocimiento; sin embargo, va más allá de éstos. El razonamiento supone poner estos argumentos en relación con situaciones por analizar o resolver, con dilemas o tareas, supone ponerlos en relación con otros conocimientos. Y éstos son procesos que no se aprenden en intercambios comunicativos lineales y unidireccionales.

Por otra parte, es necesario tener presente que ofrecer una experiencia de aprendizaje basada en la participación dialógica "chocará" inevitablemente con estructuras previamente construidas por los sujetos en sus experiencias vitales y educativas anteriores. Como he reflejado en una reciente investigación, los estudiantes universitarios no suelen participar comunicativamente en las clases por el mero hecho de darles la oportunidad de hacerlo, en dicho trabajo se pone en evidencia que la participación de los estudiantes del primer curso de un grado suele ser baja, aunque sí aumenta posteriormente a partir de la propuesta didáctica que se genere (Monarca, 2011). En este contexto, el debate y el diálogo, como sostiene Pérez Gómez (2010), se presentan como una oportunidad para la construcción, la reconstrucción y la validación de los significados personales de los sujetos.

En este sentido, y de acuerdo con lo dicho anteriormente, para que la participación sea una realidad, es necesario diseñar una propuesta didáctica que la contemple como intención educativa, para lo cual se deberán implementar las formas de enseñar apropiadas para que esto suceda. Así, la participación debe ser entendida tanto en relación con las experiencias de aprendizaje por las que se ha transitado en las trayectorias escolares previas, como en relación con las oportunidades que ofrecen las interacciones didácticas promovidas por el profesor universitario. El contemplar ambos aspectos facilitará el despliegue de prácticas educativas caracterizadas por la participación dialógica.

En este caso, el que el alumno tome la acción educativa de forma consciente, que sea partícipe de la misma, se vincula tanto con el hecho mismo de su papel dentro de los procesos que tienen lugar en el aula: el clima social de la misma y las tareas académicas (Erickson, 2003); como con la forma en que el profesor considera los saberes del alumno en tanto parte de la vida del aula, y como punto de partida para la interacción con otros conocimientos. Es necesario insistir en que esta consideración tiene una dimensión cognitiva-epistemológica vinculada a la construcción del conocimiento y la racionalidad, y otra dimensión ética 
siempre unida a la construcción del conocimiento que nos hace hablar, junto con lo anterior, de la construcción del ser, del ser en el mundo.

\section{El debate Habermas, Apel, Freire y Dussel}

En relación con lo que se está desarrollando, Habermas (1987) y Apel (1989) introducen un concepto dialógico de la ética basado en el consenso de una comunidad de comunicación que actúa sin coacciones. Aquí los autores ponen el acento en el "nosotros" que conforman la comunidad, en la cual es posible llegar a un acuerdo mediante la argumentación. Dussel (1992), por su parte, difiere de estos autores; sostiene desde su filosofía de la liberación, que lo esencial:

no es el yo o el nosotros (aun como comunidad de comunicación), o la sociedad abierta que de hecho puede cerrarse en una totalización totalitaria de la Totalidad, en su lo público burocratizado, sino el tú, el vosotros, el otro de toda comunidad de comunicación (Dussel, 1992: 72-73).

En el concepto de comunidad de iguales utilizado por Habermas (1987) y Apel (1989) no se resuelve el dilema de cómo "el otro", "no miembro", "no parte de esa comunidad", participa en la construcción de ese consenso, esa verdad; ya que al no ser parte de la comunidad, no se cumplen las condiciones por éstos enunciadas que hacen posible un consenso no coaccionado. En este sentido, la postura de Dussel (1992) resulta de sumo interés para referirse al ámbito de la acción educativa, ya que los sujetos participantes en la misma o los que de alguna manera están vinculados a ella, poseen un poder desigualmente distribuido en cuanto a las posibilidades de decidir acerca de lo que acontece en dicha acción. Este hecho definido socialmente debería ser tenido en cuenta por múltiples razones, algunas ya explicadas anteriormente, a las cuales debemos agregar la dimensión ética: no es posible quedarnos en el plano de la imposición del conocimiento, tanto por razones cognitivas ligadas a la construcción del mismo como por razones éticas vinculadas con la participación del sujeto, en este caso los estudiantes. El alumno, en la medida en que sólo reciba los efectos de las decisiones tomadas por otros, no tiene por qué asumir una responsabilidad de algo no decidido. Por tanto, si bien, incluso, el tener en cuenta al otro - en este caso el alumno-, el hacerle partícipe, también es una decisión que debe realizar la comunidad docente, es necesaria esta decisión como ámbito de posibilidades vinculadas a la construcción de la razón, del conocimiento y del sujeto en el mundo.

En esta discusión, Dussel (1992) ofrece un marco ético más amplio para comprender las relaciones posibles dentro de un intercambio comunicativo. Dentro de este marco, y atendiendo al lugar específico que el docente ocupa en dicho intercambio, el docente aparece como "responsable del otro", incluso "a su pesar", o en "su ignorancia de serlo" (Bárcena y Mélich, 2000; Levinas, 1991). Esta afirmación se fundamenta en que la acción educativa promovida por el profesor nunca acaba, o se limita, aunque quiera o lo ignore, a un contenido disciplinar concreto; éste y las formas que se proponen para que pase a formar parte de los sujetos, y otras prácticas relacionadas con este proceso (como puede ser la evaluación), forman parte del trasfondo ético.

En este proceso, de acuerdo con el trasfondo ético, como así también epistemológico, Freire (1985, 2004), al igual que Habermas (1987), Apel (1989) y Dussel (1992), insiste en el diálogo como condición de una educación para la libertad, aunque más cercano a las concepciones de éste último.

El diálogo es una exigencia existencial. Y siendo el encuentro que solidariza la reflexión y la 
acción de sus sujetos encauzados hacia el mundo que debe ser transformado y humanizado, no puede reducirse a un mero acto de depositar ideas de un sujeto en el otro, ni convertirse tampoco en un simple cambio de ideas consumadas por sus permutantes [...] Es un acto creador. De ahí que no pueda ser mañoso instrumento del cual eche mano un sujeto para conquistar a otro. La conquista implícita en el diálogo es la del mundo por los sujetos dialógicos, no la del uno por el otro. Conquista del mundo para la liberación de los hombres (Freire, 1985: 101-102).

En cualquier caso, la postura de estos cuatro autores, aunque varíe la concepción acerca de los que participan en el diálogo, se vincula siempre con un compromiso de transformación del hombre y del mundo, la implicación en un proceso continuo de mejora.

\section{Conclusión}

Este texto ha tenido la intención de ofrecer una clara argumentación a favor de la participación dialógica en los intercambios formativos de las carreras universitarias. Ésta se encuentra en la base de la construcción de un pensamiento crítico y de la formación de profesionales comprometidos con la sociedad y los demás. A continuación se resaltarán aquellas ideas principales que se desprenden de la argumentación ofrecida en las páginas precedentes:

- La participación es una práctica indispensable para la construcción de aprendizajes, para la construcción y el funcionamiento de una comunidad de aprendizaje.

- Una comunidad de aprendizaje aporta importantes beneficios para el aprendizaje y supone una forma de trabajo que constituye en sí misma una fuente de aprendizajes.

- La participación en el marco de una comunidad de aprendizaje no es sólo hablar, no es sólo opinar, es, principalmente, la interacción de los saberes que poseen los "hablantes" (conocimientos previos), con otros saberes y conocimientos contrastados.

- Dar importancia a la participación en las interacciones didácticas lleva implícita una serie de concepciones sobre: la construcción del discurso didáctico (Acosta, 2012), la construcción del conocimiento como producto social compartido, la construcción del conocimiento como proceso subjetivo, aprendizaje del sujeto, por tanto, una idea sobre cómo se aprende y cómo se enseña.

- Supone entender que la participación es una práctica y una competencia que se aprende, cuyo aprendizaje forma parte de la historia del sujeto y que, por tanto, su aprendizaje debe ser contemplado por quien enseña, lo cual necesita que forme parte de la propuesta didáctica, de la acción educativa.

- La participación, por otra parte, es entendida como valor a construir, una forma de entender la sociedad, su construcción y la importancia de la implicación del sujeto en la misma. En este sentido, la participación es la implicación activa del sujeto con sus circunstancias y las de otro, en su aquí y ahora, en el momento histórico en que vive. Supone, tal como hemos podido apreciar a través de Freire, Macedo y Dussel, asumir un compromiso con el entorno, con la sociedad y con "los otros". Por lo tanto, la participación no sólo es algo deseable y esperable, es necesaria e imprescindible.

- Vista así, la participación es una prerrequisito indispensable para desarrollar un tipo de enseñanza universitaria y un tipo de competencias para un tipo de sociedad, y a la vez un objetivo y un contenido; en la línea con la universidad que sugieren Manzano y Torrego (2009). 
- Es entender que la universidad tiene un papel importante en esta tarea, que la enseñanza universitaria tiene un compromiso con la construcción de una sociedad democrática.

- La participación, además de un fin, desde este punto de vista, es un medio, una metodología indispensable en toda propuesta didáctica; cobrando especial relevancia en la enseñanza universitaria.

- Participar es empoderar a los sujetos, futuros profesionales; empoderar a las personas como portadoras y posibles creadoras de significados y prácticas sociales y profesionales.

- La participación supone adjudicar un papel activo en la construcción social de la realidad,

\section{Referencias}

Acosta, F. (2012), "Educar, enseñar, escolarizar", en Tendencias Pedagógicas, núm. 20, pp. 93-105.

Aguilera, A., M. Mendoza, S. Racionero y M. Soler (2010), "El papel de la universidad en comunidades de aprendizaje", en Revista Interuniversitaria de Formación del Profesorado, núm. 67 (24, 1), pp. 45-56.

Apel, K. O. (1989), "La situación del hombre como problema ético", en X. Palacios y F. Jarauta (eds.), Razón, ética y politica. El conflicto de las sociedades modernas, Barcelona, Anthropos.

Bárcena, F. y J. Mélich (2000), La educación como acontecimiento ético, Barcelona, Paidós.

Bourdieu, P. y J. C. Passeron (1985), La reproducción, Barcelona, Laia.

Carreño, M. (2002), "E1 movimiento de la Escuela Nueva", en M. Carreño (ed.), Teorías e instituciones contemporáneas de educación, Madrid, Síntesis, pp. 13-43.

Coll, C. y J. Onrubia (1996), "La construcción de significados compartidos en el aula: actividad conjunta o de las realidades, del conocimiento, de los significados. La participación es acción del sujeto en el mundo, un compromiso con su construcción, con su propia emancipación y la emancipación de los otros.

Este ensayo sobre la participación dialógica en la universidad ha sido enfocado fundamentalmente desde su potencialidad como práctica pedagógica para el desarrollo del pensamiento crítico y el compromiso social. De esta manera, se ha asumido una relación imprescindible entre la formación que ofrece la universidad y su compromiso con el desarrollo de sujetos partícipes en la construcción de la "sociedad que habitan". y dispositivos semióticos en el control y seguimiento mutuo entre profesor y alumnos", en C. Coll y Edwards (eds.), Enseñanza, aprendizaje y discurso en el aula. Aproximaciones al discurso educacional, Madrid, Fundación Infancia y Aprendizaje, pp. 53-73.

Colmenar, C. (2002), "La crítica del autoritarismo escolar", en M. Carreño (ed.), Teorías e instituciones contemporáneas de educación, Madrid, Síntesis, pp. 101-129.

Dewey, J. (1995), Democracia y educación, Madrid, Morata.

Dussel,E.(1992), "Laintroducción dela transformación de la filosofía de K. O. Apel y la filosofía de la liberación (reflexiones desde una perspectiva latinoamericana)", en K. O. Apel, E. Dussel y R. Fornet, Fundamentación de la ética y la filosofia de la liberación, México, Siglo XXI-Iztapalapa, pp. 45-104. Elboj, C., I. Puigdellívol, M. Soler y S. Valls (2002), Comunidades de aprendizaje. Transformar la educación, 
Barcelona, Graó.

Erickson, F. (2003), "El discurso en el aula como improvisación: las relaciones entre la estructura de la tarea académica y la estructura de la participación social en la clase", en H. Velasco, F. J. García Castaño y A. Díaz de Rada (eds.), Lectura de antropología para educadores, Madrid, Trotta, pp. 325-353.

Flecha, R. (1997), Compartiendo palabras. El aprendizaje de las personas adultas. Barcelona, Paidós.

Freire, P. (2004), La educación como práctica de la libertad, Buenos Aires, Siglo XXI.

Freire, P. (2002), Educación y cambio, Buenos Aires, Galerna.

Freire, P. (1985), Pedagogía del oprimido, Buenos Aires, Siglo XXI.

Freire, P. y D. Macedo (1989), Alfabetización. Lectura de la palabra y lectura de la realidad, Barcelona, Paidós/ MEC.

Giner de los Ríos, F. (1924), Obras completas. Volumen $X$ : pedagogía universitaria. Problemas y noticias, Madrid, Espasa-Calpe.

Giner de los Ríos, F. (1916), Obras completas. Volumen II: la universidad española, Madrid, Espasa-Calpe.

Giroux, H. (2003), "Repensando la política de la resistencia. Nota sobre una teoría crítica de la lucha educativa", en Barbecho, Revista de Reflexión Socioeducativa, núm. 2, pp. 17-25.

Giroux, H. y P. McLaren (1998), "Lenguaje, escolarización y subjetividad: más allá de una pedagogía de la reproducción y resistencia", en $\mathrm{H}$. Giroux y P. McLaren, Sociedad, cultura y educación, Buenos Aires, Miño y Dávila, pp. 137-170.

Giroux, H. y P. McLaren (1990), "La educación del profesorado como espacio contrapúblico: apuntes para una redefinición", en Th. Popkewitz (ed.),
Formación de profesorado. Tradición. Teoría. Práctica, Valencia, Universitat de València, pp. 244-271.

Habermas, J. (1987), Teoría de la acción comunicativa I, Madrid, Taurus.

Kemmis, S. (1999), "La investigación-acción y la política de la reflexión", en A. Pérez, J. Barquín y J. F. Angulo (eds.), Desarrollo profesional del docente. Política, investigación y práctica, Madrid, Akal, pp. 95-118.

Levinas, L. (1991), Ética e infinito, Madrid, Visor.

Loroño, M., M. J. Alonso-Olea y I. MartínezDomínguez (2010), "La metodología dialógica en las aulas universitarias", en Revista de Educación, núm. 352, pp. 309-329.

Luzuriaga, L. (1968), Pedagogía social y política, Buenos Aires, Losada.

Manzano, V. y L. Torrego, (2009), "Tres modelos para la universidad", en Revista de Educación, núm. 350, pp. 447-489.

Monarca, H. (2011), "Evaluación de la participación de los estudiantes en el nuevo grado de primaria", en Revista Interuniversitaria de Formación del Profesorado, núm. 38 (14), pp. 105-115.

Pérez Gómez, A. (2010), "Aprender a educar. Nuevos desafíos para la formación de docentes", en Revista Interuniversitaria de Formación del Profesorado, núm. 68 (24, 2), pp. 37-60.

Ramírez-Romero, J. L. y N. A. Quintal-García (2011), “¿Puede ser considerada la pedagogía crítica como una teoría general de la educación?", en Revista Iberoamericana de Educación Superior (RIES), vol. II, núm. 5, pp. 114-125, http://ries.universia. net/index.php/ries/article/view/141 [consulta: abril de 2012].

Young, R. (1993), Teoría crítica de la educación y discurso en el aula, Barcelona, Paidós/MEC.

\section{Cómo citar este artículo:}

Monarca, Héctor (2013), "Participación dialógica en la universidad: condición para el desarrollo del pensamiento crítico y el compromiso social", en Revista Iberoamericana de Educación Superior (RIES), México, UNAM-IISUE/Universia, Vol. IV, núm. 9, pp. 53-62, http://ries.universia.net/index.php/ries/article/view/305/html_40 [consulta: fecha de última consulta]. 